Chen, Q. And Zhang, Z. 2005. “Prediction of particle transport in enclosed environment,”

China Particuology, 3(6), 364-372.

\title{
PREDICTION OF PARTICLE TRANSPORT IN ENCLOSED ENVIRONMENT
}

\author{
Qingyan Chen* and Zhao Zhang \\ Air Transportation Center of Excellence for Airliner Cabin Environment (ACER), School of Mechanical Engineering, \\ Purdue University, West Lafayette, IN 47907, USA \\ *Author to whom correspondence should be addressed: E-mail: yanchen@purdue.edu
}

\begin{abstract}
Prediction of particle transport in enclosed environment is crucial to the welfare of its occupants. The prediction requires not only a reliable particle model but also an accurate flow model. This paper introduces two categories of flow models - Reynolds Averaged Navier-Stokes equation modeling (RANS modeling) and Large Eddy Simulation (LES); as well as two popular particle models - Lagrangian and Eulerian methods. The computed distributions of air velocity, air temperature, and tracer-gas concentration in a ventilated room by the RANS modeling and LES agree reasonably with the experimental data from the literature. The two flow models gave similar prediction accuracy. Both the Lagrangian and Eulerian methods were applied to predict particle transport in a room. Again, the computed results were in reasonable agreement with the experimental data obtained in an environmental chamber. The performance of the two methods was nearly identical. Finally the flow and particle models were applied to study particle dispersion in a Boeing 767 cabin and in a small building with six rooms. The computed results look plausible.
\end{abstract}

Keywords computational fluid dynamics, Lagrangian method, Eulerian method

\section{Introduction}

In developed countries, people spend more than $90 \%$ of their time in enclosed environments, such as buildings and transportation vehicles. The air quality in the enclosed environments is therefore an important factor of their welfare. The air quality is determined by the level of air contaminants, such as materials used for internal furnishings, equipment, and cleaning, personal activities, environmental tobacco smoke, pesticide, furnaces, soil emissions, and combustion products from cooking, as well as those from outdoors due to infiltration, such as traffic pollutants, pollen, dusts, etc. Many of the pollutants are suspended particles in air, such as dusts, smoke, fumes, and mists (ASHRAE, 2005). Wallace (1996) showed that people who were exposed to micron-sized particulate matter was related to these environments. In addition, the terrorist attacks on September 11, 2001 and the following anthrax dispersion by mails have spawned concerns about various possible forms of terrorism, including airborne/aerosolized chemical and biological warfare agent attacks. The study of particle transport in enclosed environments has thus received more attention at present.

The study of particle transport in enclosed environments can be preformed by experimental measurements and computer simulations. Experimental measurements that are often regarded as reliable are expensive and sometimes can be dangerous, such as for SARS, bird influenza, and anthrax transport. Computer simulations are a good alternative. Since many approximations are used in the computer models, experimental validation of the simulated results is necessary. This paper provides a general overview of using computer models to simulate micron-sized particle transport in enclosed environment and the assessment of the model performance through a few examples.

\section{Computer Models}

The most popular computer model for studying particle dispersion in enclosed environment is computational fluid dynamics (CFD). CFD has become an indispensable tool for gathering information to be used for design, control and optimization of enclosed environments. To accurately predict micro-sized particle transport, the first step is to determine the airflow pattern with acceptable precision.

CFD can be divided into direct numerical simulation, Large Eddy Simulation (LES), and the Reynolds Averaged Navier-Stokes equations with turbulence models (RANS modeling). Direct numerical simulation would require a fast computer that does not currently exist, and would take years of computing time for predicting air distributions in enclosed environment. Only LES and RANS modeling are appropriate for studying airflow in enclosed environment. 


\subsection{RANS modeling}

RANS modeling separate all spatial parameters, such as velocity and temperature, into their mean and fluctuating components and the fluctuating components are only predicted with time-averaged rootmean-square (rms) values. Thus, RANS modeling solves only the mean components. For time-averaged and incompressible buoyant flow, the basic RANS equations are

(a) Continuity equation:

$\frac{\partial\left(\rho U_{i}\right)}{\partial x_{i}}=0$

(b) Momentum equations:

$\frac{\partial\left(\rho U_{i}\right)}{\partial t}+\frac{\partial\left(\rho U_{j} U_{i}\right)}{\partial \mathbf{x}_{j}}=\frac{\partial}{\partial \mathbf{x}_{j}}\left(v_{\text {eff }} \frac{\partial U_{i}}{\partial \mathbf{x}_{j}}\right)+S_{U_{i}}$

$S_{u i}$ is the source term and $v_{\text {eff }}$ the effective viscosity. $v_{\text {eff }}$ has the form:

$v_{\text {eff }}=v_{t}+v$

$v_{t}$ is the eddy (turbulent) viscosity. In the standard k- $\varepsilon$ model (Launder and Spalding, 1974) that is most popular, the eddy viscosity is obtained from

$v_{\mathrm{t}}=\mathrm{C}_{\mu} \frac{\mathrm{k}^{2}}{\varepsilon}$

Then we need two extra transport equations to solve turbulent kinetic energy, $\mathrm{k}$, and its dissipation rate, $\varepsilon$.

(c) Turbulent kinetic energy equation:

$\frac{\partial(\rho k)}{\partial t}+\frac{\partial(\rho U k)}{\partial \mathbf{x}_{j}}=\frac{\partial}{\partial \mathbf{x}_{j}}\left(\rho \frac{v_{t}}{\sigma_{k}} \frac{\partial k}{\partial \mathbf{x}_{j}}\right)+\rho\left(G_{k}+G_{b}-\varepsilon\right)$

(d) Dissipation rate of turbulent kinetic energy equation:

$\frac{\partial(\rho \varepsilon)}{\partial \mathrm{t}}+\frac{\partial\left(\rho \bigcup_{j} \varepsilon\right)}{\partial \mathbf{x}_{j}}=\frac{\partial}{\partial \mathbf{x}_{j}}\left(\rho\left(v+\frac{v_{t}}{\sigma_{\varepsilon}}\right) \frac{\partial \varepsilon}{\partial \mathbf{x}_{j}}\right)+\rho \frac{\varepsilon}{k}\left(C_{1 \varepsilon} G_{k}+C_{1 \varepsilon} C_{3 \varepsilon} G_{b}-C_{2 \varepsilon} \varepsilon\right)$

where the shear production term is defined by

$$
G_{k}=v_{t}\left(\frac{\partial U_{i}}{\partial x_{j}}+\frac{\partial U_{j}}{\partial x_{i}}\right) \frac{\partial U_{i}}{\partial x_{j}}
$$

and, if heat transfer is involved, for ideal gas, the buoyant production term by

$G_{b}=-\frac{v_{t} g_{i}}{\rho_{\sigma_{T}}} \frac{\partial \rho}{\partial x_{i}}$

\subsection{Large eddy simulation}

LES is based on Navier-Stokes and mass continuity equations. LES assumes that flow motion can be separated by large and small scale eddies through a filter. The large scale eddies are directly solved in LES, while the smaller scales are modeled. Since larger scale eddies carry the majority of the energy, they are more important. The smaller scales have been found to be more universal, and hence are more easily modeled. By filtering Navier-Stokes and mass continuity equations, one would obtain the governing equations for the large-eddy motions as

(a) Continuity equation:

$\frac{\partial \overline{u_{i}}}{\partial \mathbf{x}_{i}}=0$

(b) Momentum equations:

$\frac{\partial \overline{u_{i}}}{\partial t}+\frac{\partial}{\partial x_{j}}\left(\overline{u_{i}} \cdot \overline{u_{j}}\right)=-\frac{1}{\rho} \frac{\partial \bar{p}}{\partial x_{i}}+v \frac{\partial^{2} \bar{u}_{i}}{\partial x_{i} \partial x_{j}}-\frac{\partial \tau_{i j}}{\partial x_{j}}$

The bar represents grid filtering. For example, a one-dimensional filtered velocity can be obtained from

$\overline{u_{i}}=\int G\left(x, x^{\prime}\right) u_{i}(x) d x^{\prime}$

where $G\left(x, x^{\prime}\right)$, the filter kernel, is a localized function. $G\left(x, x^{\prime}\right)$ is large only when $\left(x-x^{\prime}\right)$ is less than a length scale or a filter width. The length scale is a length over which averaging is performed. Flow eddies larger than the length scale are "large eddies" and smaller than the length scale are "small eddies". If a box filter is used: 
$\mathrm{G}\left(\mathrm{x}_{\mathrm{i}}\right)= \begin{cases}\frac{1}{\Delta_{\mathrm{i}}} & \left(\left|\mathrm{x}_{\mathrm{i}}\right| \leq \frac{\Delta_{\mathrm{i}}}{2}\right) \\ 0 & \left(\left|\mathrm{x}_{\mathrm{i}}\right|>\frac{\Delta_{\mathrm{i}}}{2}\right)\end{cases}$

where $\Delta_{\mathrm{i}}$ is the filter width.

The subgrid-scale Reynolds stresses in Eq. (10),

$\tau_{\mathrm{ij}}=\overline{\mathrm{u}_{\mathrm{i}} \mathrm{u}_{\mathrm{j}}}-\overline{\mathrm{u}}_{\mathrm{i}} \cdot \overline{\mathrm{u}}_{\mathrm{j}}$

are unknown and must be modeled. The simplest but also most popular one uses the Smagorinsky subgrid-scale model (Smagorinsky, 1963) to model the subgrid-scale Reynolds stresses. The model has been widely used since the pioneer work by Deardorff (1970). The Smagorinsky model assumes that the subgrid-scale Reynolds stresses, $\tau_{i j}$, are proportional to the strain rate of the tensor,

$$
\begin{aligned}
\overline{\mathrm{S}}_{\mathrm{ij}} & =\frac{1}{2}\left(\frac{\partial \overline{\mathrm{u}}_{\mathrm{i}}}{\partial \mathrm{x}_{\mathrm{j}}}+\frac{\partial \overline{\mathrm{u}}_{\mathrm{j}}}{\partial \mathrm{x}_{\mathrm{i}}}\right) \text {, namely } \\
\tau_{\mathrm{ij}} & =-2 \mathrm{v}_{\mathrm{SGS}} \overline{\mathrm{S}}_{\mathrm{ij}}
\end{aligned}
$$

where $v_{\text {SGS }}$ is the subgrid-scale eddy viscosity defined as

$v_{\mathrm{SGS}}=\left(\mathrm{C}_{\mathrm{SGS}} \Delta\right)^{2}\left(2 \overline{\mathrm{S}_{\mathrm{ij}}} \cdot \overline{\mathrm{S}_{\mathrm{ij}}}\right)^{\frac{1}{2}}$

where $\mathrm{C}_{\mathrm{SGS}}=0.1 \sim 0.2$ is the Smagorinsky constant, which varies according to flow type. The Smagorinsky model actually adopts the mixing length model of RANS modeling to the subgrid-scale model of LES.

LES has been a very useful tool in simulating flow in multiple scales. It was developed for application in meteorology. In early 1970s, the grid resolution was as large as $100 \mathrm{~km}$. Today, the same application is with a grid resolution as fine as one kilometer. On the other end of spectrum, LES has no limitation on the size of small scale. When LES has a scale that can catch the smallest size of turbulent flow, it turns to direct numerical simulation. On the other hand, RANS modeling is applicable to certain scales. Different turbulent models should be used for different scales of flow. It is also possinle to mix the LES and RANS modeling together.

Regardless if RANS modeling or LES is employed, a particle model must be used to calculate particle transport. There are two generic approaches for the numerical simulation of particle transport in airflows: the Lagrangian method and the Eulerian method. In the Lagrangian method (Loth, 2000), the velocity, mass, and temperature history of each particle in the cloud are calculated. The local cumulative motion and state of each particle in the cloud represent the spatial properties of the cloud. In the Eulerian approach, the cloud of particles is considered to be a second fluid that behaves like a continuum, and equations are developed for the average properties of the particles in the cloud (Crowe et al., 1998). Each approach has its advantages and disadvantages depending on the nature of the flow. Compared to the Eulerian method, the main drawback of the Lagrangian approach is that a large amount of particles must be injected into the flow field in order to obtain statistically independent results. However, the Lagrangian does not need a diffusion coefficient of the particles. The following two sections will briefly describe the two methods normally used in enclosed environments.

\subsection{Lagrangian method}

In most cases, the particle concentration in enclosed environment is relatively low and the size is small. The interaction between the carrier air and the particles has been treated as a one-way coupling, assuming that the effect of particles on the turbulent flow is negligible due to low solid loading and relatively small particle settling velocity, and that particles do not coagulate.

The Lagrangian method computes the trajectory of each particle by solving the momentum equation based on Newton's second law,

$\frac{\mathrm{d}\left(\mathrm{m}_{\mathrm{P}} \mathrm{v}_{\mathrm{i}}\right)}{\mathrm{dt}}=\sum \mathrm{F}_{\mathrm{i}}$

with

$\frac{d X_{i}}{d t}=v_{i}$ 
Momentum force is transferred between air and particles through inter-phase drag and lift forces, which can be divided into, but not limited to, the following parts: the drag force, pressure gradient force, unsteady forces which include Basset force and virtual mass force, Brownian force, and body force, such as gravity force and buoyancy force (Crowe et al., 1998). For particles with a certain size and density, some of the forces could be very small compared to others, and thus being neglected.

Here we consider only solid particles and assume the particle shape to be sphere. We further limit our study to the particles with diameter ranging from 0.1 to $20 \mu \mathrm{m}$ and the density is at the order of $10^{3} \mathrm{~kg} / \mathrm{m}^{3}$, which are mostly common in enclosed environments. As analyzed by Crowe, et al. (1998), the pressure gradient force can be neglected. Furthermore, according to the calculations of Hjelmfelt and Mockros (1966), the Basset force and virtual mass force become insignificant for such a particle density in air. Thus, these two unsteady forces can also be neglected.

If the size of a particle suspended in a fluid is very small (less than $0.1 \mu \mathrm{m}$ ), the discrete nature of molecular motion affects the motion of the particle, exhibiting a random motion due to collisions of molecules with particles. This is called Brownian motion. For super micron particles, the Brownian force is not significant. Therefore, only drag force, gravity force, buoyancy force (and Saffman lift force for the ventilated cavity) are normally considered in most studies for enclosed environment. So the term on the right side in Eq. (16) can be expanded as

$\sum \mathrm{F}_{\mathrm{i}}=\mathrm{F}_{\text {DRAG } \mathrm{i}}+\mathrm{F}_{\text {GRAVi }}\left(+\mathrm{F}_{\mathrm{SAF} i}+\mathrm{F}_{\mathrm{bi}}\right)$

When studying particle motion in airflows, one of the most important forces is the drag force, which acts on the particle in a uniform pressure field when there is no acceleration of the relative velocity between the particle and the conveying fluid. The force is quantified by the drag coefficient, $C_{D}$, through the equation

$$
F_{\text {DRAGi }}=-C_{D} \frac{\pi}{8} \rho d_{P}^{2}|\vec{u}-\vec{v}|\left(v_{i}-u_{i}\right)
$$

In general, the drag coefficient will depend on the particle shape and orientation with respect to the flow as well as on the flow parameters such as Reynolds number, Mach number, turbulence level, etc. Hinds (1982) computed the drag coefficient for a spherical solid particle with

$\mathrm{C}_{\mathrm{D}}=\max \left(0.44, \mathrm{C}_{\mathrm{D}}\right.$ of Eq. 21$)$

$$
C_{D}=\frac{24.0\left(1.0+0.15 \operatorname{Re}_{\mathrm{P}}^{0.687}\right)}{\operatorname{Re}_{\mathrm{P}}}
$$

where $\mathrm{Re}_{\mathrm{p}}$ is the particle Reynolds number :

$\operatorname{Re}_{\mathrm{P}}=\frac{|\overrightarrow{\mathrm{u}}-\overrightarrow{\mathrm{v}}| \mathrm{d}_{\mathrm{P}}}{v}$

The body forces in enclosed environment are the gravity and the buoyancy forces, which can be expressed as

$F_{\text {GRAVi }}=\left(\rho_{P}-\rho\right) \frac{\pi}{6} d_{p}^{3} g_{i}$

The shear lift force derived by Saffman (1965) is the same as in McLaughlin (1989):

$F_{\text {SAF }}=K \frac{\pi}{3} \sqrt{v} d_{P}^{2} \rho \frac{d_{i j}}{\left(d_{k l} d_{k l}\right)^{1 / 4}}\left(u_{j}-v_{j}\right)$

where $\mathrm{K}=2.594$ and $\mathrm{d}_{\mathrm{ij}}=\frac{1}{2}\left(\frac{\partial \mathrm{u}_{\mathrm{i}}}{\partial \mathrm{x}_{\mathrm{j}}}+\frac{\partial \mathrm{u}_{\mathrm{j}}}{\partial \mathrm{x}_{\mathrm{i}}}\right)$. Substituting Eqs. (19), (23) and (24) into Eq. (16) and dividing by the mass of particles, $m_{p}$, on both sides, one can obtain the particle motion equation:

$$
\begin{aligned}
\frac{d v_{i}}{d t} & =-\frac{3}{4} \frac{\rho C_{D}}{d_{p} \rho_{p}}|\vec{u}-\vec{v}|\left(v_{i}-u_{i}\right)+\left(\rho_{p}-\rho\right) \frac{\pi}{6} d_{p}^{3} g_{i}+\left(1-\frac{\rho}{\rho_{p}}\right) g_{i}\left(+\frac{2 k \rho \sqrt{v} d_{i j}}{\rho_{p} d_{p}\left(d_{l k} d_{k l}\right)^{1 / 4}}\left(u_{j}-v_{j}\right)\right) \\
& =-f_{p}\left(v_{i}-u_{i}\right)+\left(\rho_{p}-\rho\right) \frac{\pi}{6} d_{p}^{3} g_{i}+\left(1-\frac{\rho}{\rho_{p}}\right) g_{i}\left(+\frac{2 K \rho \sqrt{v} d_{i j}}{\rho_{p} d_{p}\left(d_{l k} d_{k l}\right)^{1 / 4}}\left(u_{j}-v_{j}\right)\right)
\end{aligned}
$$

where:

$f_{p}=\frac{3}{4} \frac{\rho C_{D}}{d_{p} \rho_{p}}|\vec{u}-\vec{v}|$

is the response "frequency" of the particle to changes in flow conditions surrounding the particle. 
For submicron particles, the Brownian force can be also included depending on its significance. It can be expressed as (Li and Ahmadi, 1992):

$F_{b i}=\zeta_{i} \sqrt{\frac{216 \rho v \sigma T}{\pi \rho_{p}^{2} d_{p}^{5} C_{c} \Delta t}}$

where $\sigma=1.38 \times 10^{-23} \mathrm{~J} / \mathrm{K}$ is the Boltzmann constant, $\zeta$ a Gaussian random number, $\Delta \mathrm{t}$ the time step for particle tracking, $\mathrm{C}_{\mathrm{c}}$ the Cunningham correction factor, and $\mathrm{T}$ the ambient air temperature.

Since LES gives transient flow field, the Lagrangian method can easily consider the turbulent effect on particle trajectories. However, RANS modeling solves only averaged flow field so the particle turbulent dispersion needs to be modeled. One of the most popular approaches is the use of the discrete random walk model (DRW). It correlates the particle turbulent dispersion with the flow turbulent kinetic energy:

$\mathrm{u}_{\mathrm{i}}^{\prime}=\zeta \sqrt{2 \mathrm{k} / 3}$

where $u_{i}^{\prime}$ is instantaneous air velocity, $\zeta$ is a Gaussian random number, $k$ is the turbulent kinetic energy. By modeling the transient effect of airflow through $\mathrm{k}$ and $\zeta$, the particle is then in a modeled transient state and possesses stochastic behaviors.

\subsection{Eulerian method}

The complexity of Eulerian modeling depends on particle's physical characteristics and its volume fraction in the air. As stated previously that particles in enclosed environment are small and dilute, the Eulerian method often assumes the particle phase as a scalar species and the particle phase follows the passive scalar transport equation:

$\frac{\partial \rho \mathrm{C}}{\partial \mathrm{t}}+\frac{\partial}{\partial \mathbf{x}_{\mathrm{i}}}\left(\rho \overline{\mathrm{u}_{\mathrm{i}}} \mathrm{C}-\Gamma \frac{\partial \mathrm{C}}{\partial \mathbf{x}_{\mathrm{i}}}\right)=\mathrm{S}_{\mathrm{C}}$

where $C$ is the particle concentration, $\Gamma$ the effective particle diffusion coefficient, and $S_{c}$ the particle source term. The particle diffusion coefficient has the form:

$\Gamma=\rho\left(v_{\mathrm{p}}+\mathrm{D}\right)$

where $v_{p}$ is particle turbulent diffusivity and $D$ the Brownian diffusivity of particles. When particle size is larger than $0.01 \mu \mathrm{m}$, the Brownian diffusivity is negligible compared with turbulent diffusivity. Since the current study focus on larger particles $(0.1 \sim 10 \mu \mathrm{m})$, only turbulent diffusivity was important. Hinze (1975) proved mathematically that, for long diffusion times, this particle turbulent diffusivity equals to the turbulent viscosity of the flow in homogeneous flow fields:

$v_{\mathrm{p}}=v_{\mathrm{t}}$

Although the complex airflow in enclosed environment is not exactly homogeneous, this equality still holds due to small particle relaxation time in the environment (Lai and Nazaroff, 2000).

The particle source term includes both physical sources and numerical sources. The physical sources are real ones that can generate particles inside domain. The numerical sources occur when Eq. (29) is modified to represent certain mechanism. The modifications are usually written in form of source terms so that the left hand side of Eq. (29) remains unchanged. By doing this, the scalar equation then keeps the same general form as the momentum, energy and turbulent equations (Patankar, 1980) and the CFD can solve all equations simultaneously with the same algorithm. In indoor applications, for instance, the gravitational settling is often considered for coarse particles by adding the drift term as a numerical source (Zhao et al., 2004).

\section{Examples of Applications}

In this section, we will evaluate the performance of RANS modeling and LES as well as the Lagrangian and Eurelian methods by applying them to ventilated rooms where experimental data are available. Then this investigation will show two applications of the flow and particle models.

\subsection{Evaluation of RANS modeling and large eddy simulation}

The evaluation of the performance of the airflow models for enclosed environments was done by applying the models to predict airflow in a ventilated room as shown in Fig. 1(a). The room was 5.16 $\mathrm{m}$ long, $3.65 \mathrm{~m}$ wide, and $2.43 \mathrm{~m}$ high. The room simulated a two-person office with many heated and unheated objects, such as the occupants, lighting, computers, and furniture. Yuan et al. (1999) 
measured the airflow pattern and the distributions of air temperature, air velocity, and tracer-gas $\left(\mathrm{SF}_{6}\right)$ concentration in the room. The tracer-gas was used to simulate $\mathrm{CO}_{2}$ emissions from the two occupants. In the room, cold air was supplied through the diffuser in the lower part of a room, and warm air was extracted at the ceiling level. The temperature of the inlet airflow from the diffuser was $17.0^{\circ} \mathrm{C}$, and the ventilation rate was $0.053 \mathrm{~m}^{3} / \mathrm{s}$. The total heat sources from the occupants, computers, and lighting in the room were $636 \mathrm{~W}$. The tracer gas (SF6) sources released at the head of two occupants were with a total flow rate of $80 \mathrm{ml} / \mathrm{h}$.
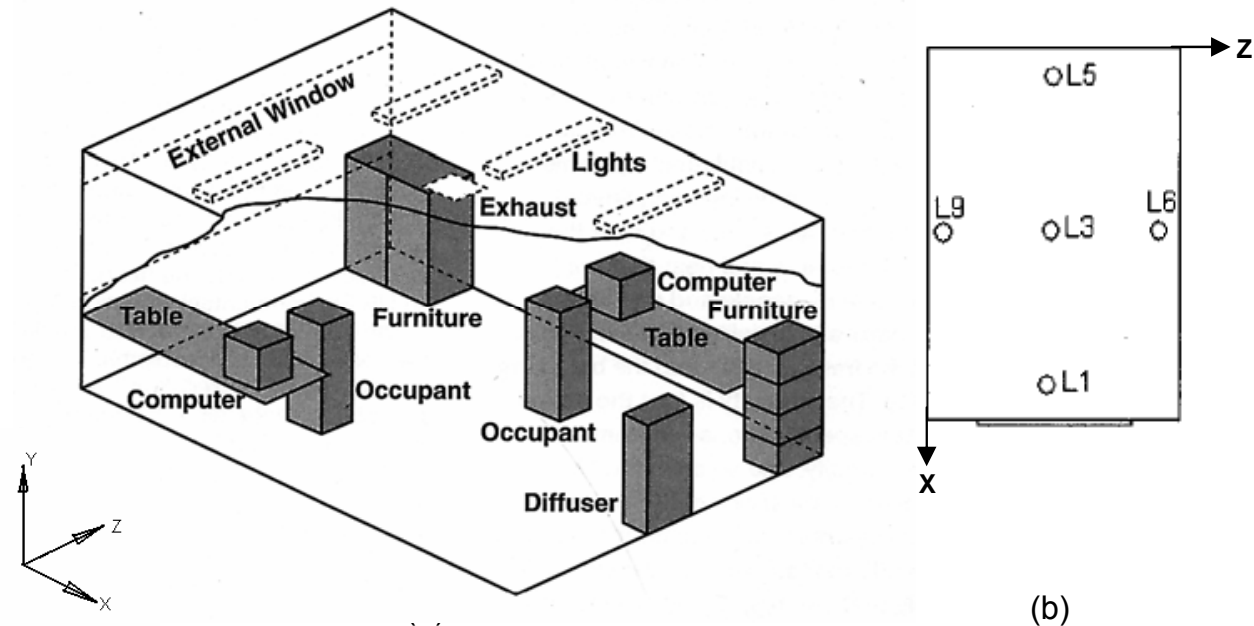

FIg. 1. (a) Chamber schematic and (b) the positions where measured data are selected

Although Yuan et al. (1999) measured the airflow and the distributions of air velocity, air temperature, and $\mathrm{SF}_{6}$ concentration in many locations, this study selected the data measured in five locations as shown in Fig. 1(b) to evaluate the RANS and LES models. Figure 2 compares the computed results with the measured data in the five positions selected. The horizontal coordinate represents normalized air velocity, air temperature, and $\mathrm{SF}_{6}$. The vertical coordinate shows the height normalized by the room height.

11

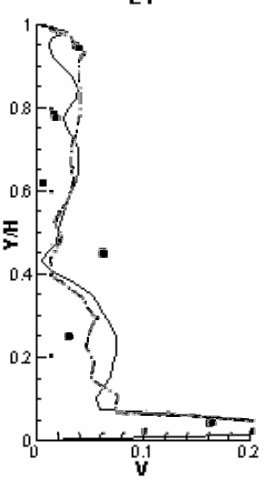

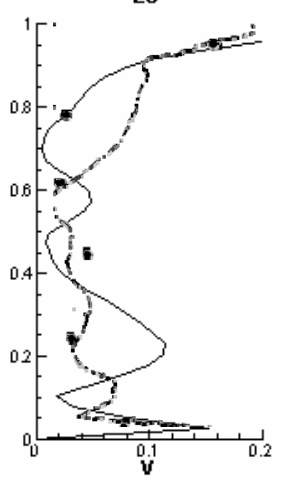

L5

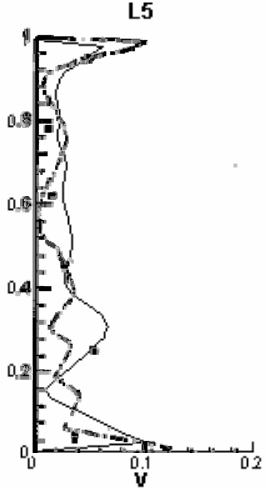

(a) Air velocity
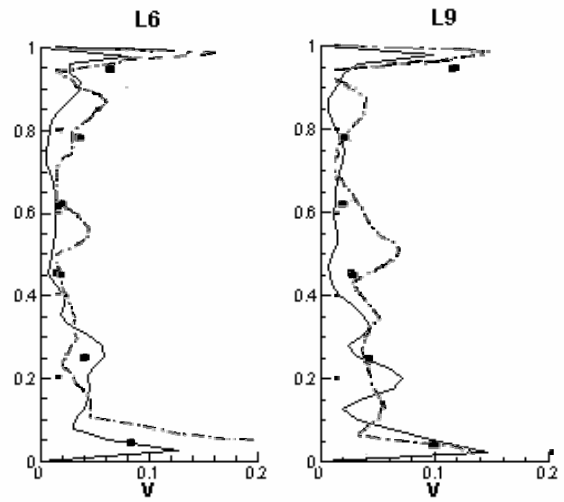
L1

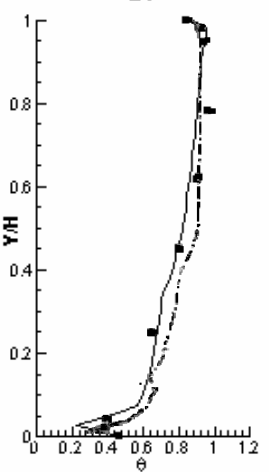

11

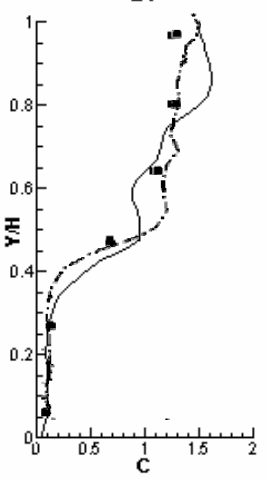

L3
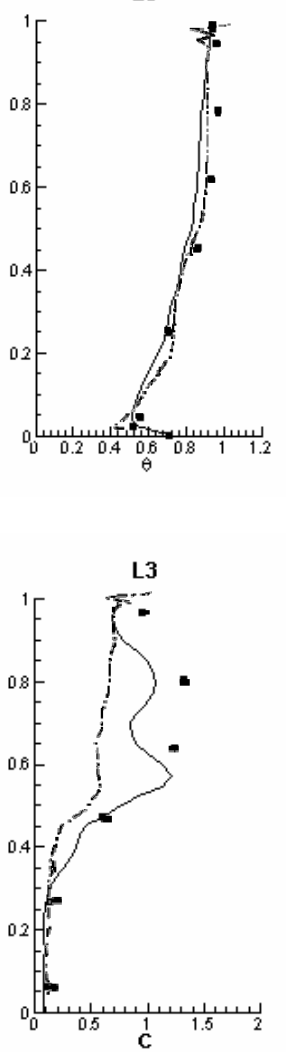

L5

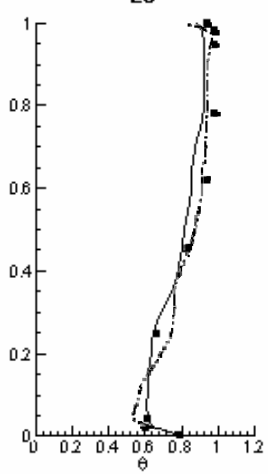

(b) Air temperature

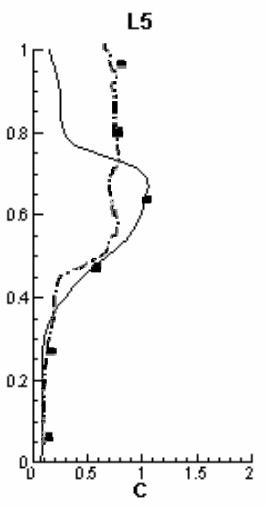

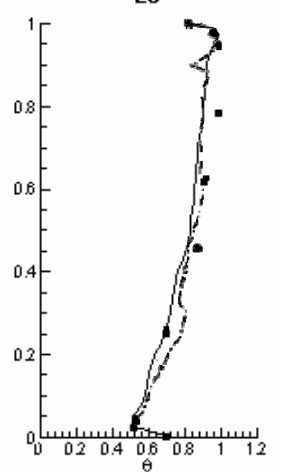

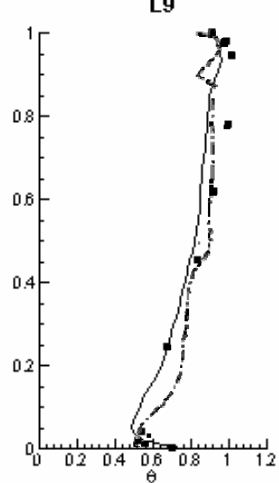

(c) $\mathrm{SF}_{6}$ concentration

L9
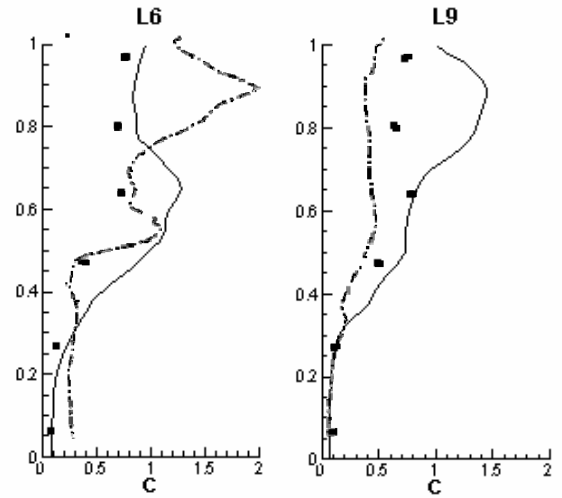

Fig. 2. Comparison of the computed air velocity, air temperature and $S F_{6}$ concentrations with the measured data for the room (Symbols - measurement data, solid lines - RANS simulations, and dash dot lines - LES simulations)

It is very difficult to tell from Fig. 2 whether the RANS modeling or the LES performed better in predicting the distributions of air velocity, air temperature, and $\mathrm{SF}_{6}$ concentration. When the air velocity is low as in most enclosed environments, the measurements of air velocity have a great uncertainty. The differences between the computed results and measured data are within the measurement errors. As shown in Fig. 2(b), both the RANS modeling and LES can predict very well the air temperature profiles. On the other hand, it is relatively easy and more accurate to measure air temperature. The agreement between the calculated temperatures and measured data is very good. However, there were great uncertainties in the calculations of $\mathrm{SF}_{6}$ distributions. Although one can often analog temperature and concentration calculations, the RANS modeling and LES did not give similar predictions. Although the trend of the $\mathrm{SF}_{6}$ profiles can be captured, the differences between the two methods are significantly. The experiment has also shown unstable distributions of $\mathrm{SF}_{6}$ concentration. The $\mathrm{SF}_{6}$ concentration monitored in a specific location in the room can fluctuate dramatically within a minute or two. It took about 30 seconds to measure $\mathrm{SF}_{6}$ concentration at a specific location through sampling. Thus, very similar to air velocity, it is difficult to tell whether the RANS modeling, LES, or the experiment can be more trusted. Nevertheless, both RANS modeling and LES could be used for engineering applications because the applications normally use RANS modeling or LES to do parametric studies. Both the RANS modeling and LES can certainly predict trends of changes.

It is important to note that the results from LES are more informative and look more realistic. The LES provides not only the mean values of the parameters, such as velocity, temperature, pressure, and species concentrations, but also the changes of the parameters over time. As flow motion is in multiple scale, LES presents a powerful tool to study the flow mechanism in multiple scales. The LES contains only one or no empirical coefficient in the model, it is less artificial than RANS modeling. It is more meaningful. 


\subsection{Evaluation of the Lagrangian and Eulerian methods}

With the reasonable accuracy of RANS modeling and LES in calculating air distributions, we could further evaluate the performance of the Lagrangian and Eulerian methods in predicting the transports of particles in enclosed environment. Since very little experimental data are available from literature concerning particle dispersion in enclosed environment, we have conducted experimental measurements in an environmental chamber as shown in Fig. 3 (Zhang and Chen, 2005). The chamber dimensions in the $\mathrm{x}, \mathrm{y}$, and $\mathrm{z}$-directions were $4.91 \mathrm{~m}, 2.44 \mathrm{~m}$, and $4.31 \mathrm{~m}$, respectively. Two air supply openings were located on the floor, and the exhaust outlet was located on the ceiling. Air was supplied to the room at a total airflow rate of $0.0944 \mathrm{~m}^{3} / \mathrm{s}$. Four heated human simulators were placed on the floor and six lamps were installed on the ceiling.
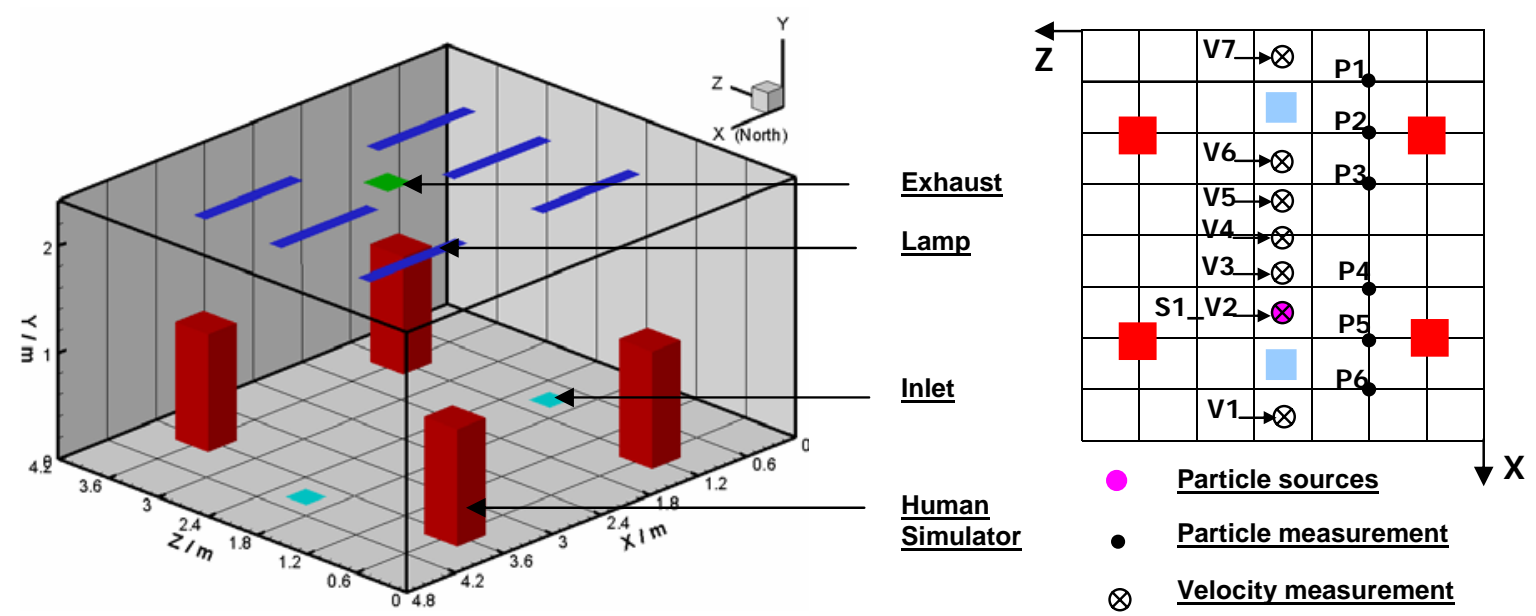

Fig. 3. Schematic of the environmental chamber and measurement locations

Forty-nine anemometers were installed at seven different heights in seven different locations along the center plane of the room to simultaneously measure air velocity and air temperature. For this case, only a RANS model was used and the agreement between the computed and measured air velocity and temperature is comparable to that found in the case reported in Section 3.1.

A condensation mono-disperse aerosol generator was used to generate particles of $0.7 \mu \mathrm{m}$ with a density of $912 \mathrm{~kg} / \mathrm{m}^{3}$. As shown in Fig. 3, the particle source was located at position $\mathrm{S} 1,0.3 \mathrm{~m}$ above the floor. Particles were introduced into the room at a flow rate of $7 \times 10^{-5} \mathrm{~m}^{3} / \mathrm{s}(250 \mathrm{l} / \mathrm{h})$. In the experiment, the nominal concentration (steady-state concentration at the exhaust) was on the order of $10^{9}$ particles $/ \mathrm{m}^{3}$. Since the concentration was two magnitudes higher than the background concentration, the background noise in the measurement data was negligible. Particle concentrations were measured in five different heights at the six positions shown in the right part of Fig. 3.

The present simulations used a structured grid containing a total of 389,338 control volumes. The concentration calculation by the Lagrangian method used a uniform mesh throughout the domain, containing $24 \times 12 \times 26=7,488$ cells. Since the particle size is less than $1 \mu \mathrm{m}$, Brownian force became more significant and a direct neglect may not be appropriate. So the Brownian force was considered in the study. Since the Lagrangian modeling involved stochastic process, the stability of concentration calculation was studied (Zhang and Chen, 2005). It was concluded that 10,000 trajectories were necessary for such a problem and 10,000 trajectories were used.

Fig. 4 shows the comparison between simulated results by the Lagrangian and Eulerian methods and the measured particle concentration in the room. The particle concentration is normalized by the steady-state nominal particle concentration at the exhaust. The computed particle concentration profiles by the Lagrangian and Eulerian methods agree well with the experimental data. The Eulerian method performed slightly better for position 6 . However, the Lagrangian method provided also temporal distributions of particles while the Eulerian method gave only concentration. The Lagrangian method allows us to track the motion and changes for each individual particle. This implies that the method is more useful for multiple-scale studies. On the other hand, the Eulerian method gives only 
the global value and uses an empirical diffusion coefficient. Thus, it may not be able to give the information one would need for multiple-scale studies.

Very similar to the results for air velocity, temperature and $\mathrm{SF}_{6}$ concentration in the previous case, the Lagrangian and Eulerian methods can predict particle distributions in enclosed environment for engineering applications with reasonable accuracy.
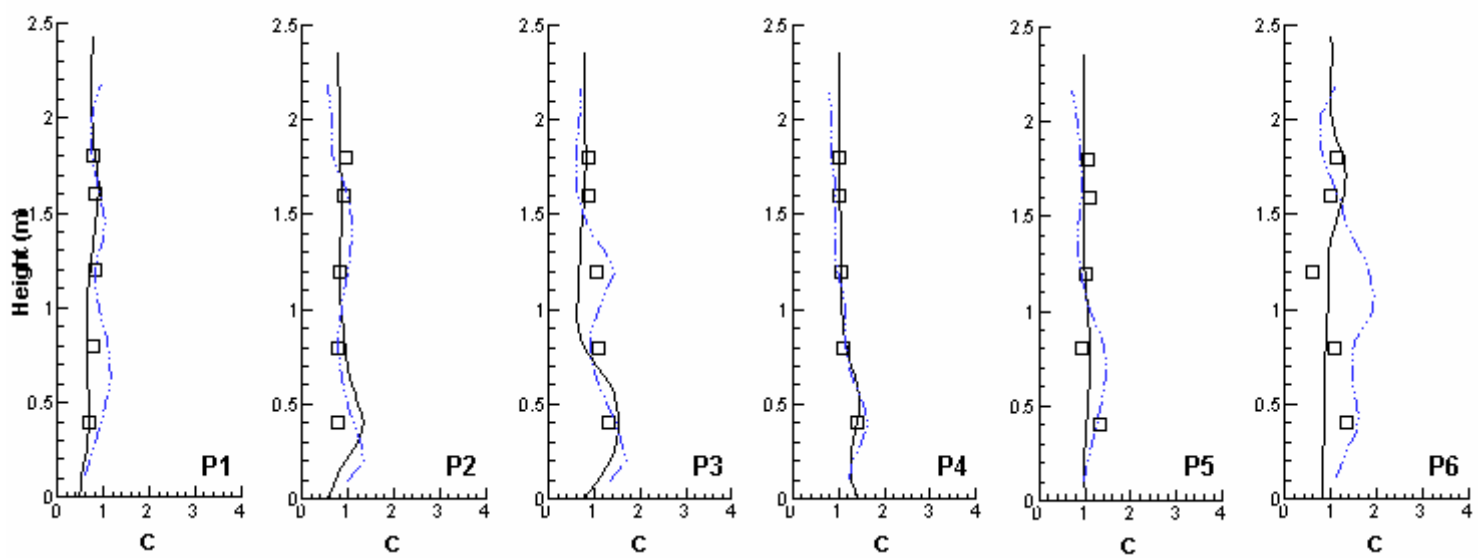

Fig. 4. Comparison of computed particle concentration profiles with experimental data for a particle source at S1 (Rectangular symbols - experimental data, dash-dot-dot lines - Lagrangian method, and solid lines - Eulerian method)

\subsection{Application of RANS modeling with Lagrangian method to particle dispersion in an aircraft cabin}

With the validated RANS modeling and Lagrangian method, this section shows a practical application of the computer modeling. With the fresh memory of SARS outbreak in 2003 and the new threat of bird flu those days, it has been a great concern of infectious disease transmission in public transportation. The situation in a commercial airliner cabin is especially worse due to limited space and mobility. Hence, we applied the RANS modeling and Lagrangian method to predict liquid droplet dispersion due to coughing of a passenger in a Boeing 767 cabin as shown in Fig. 5(a).

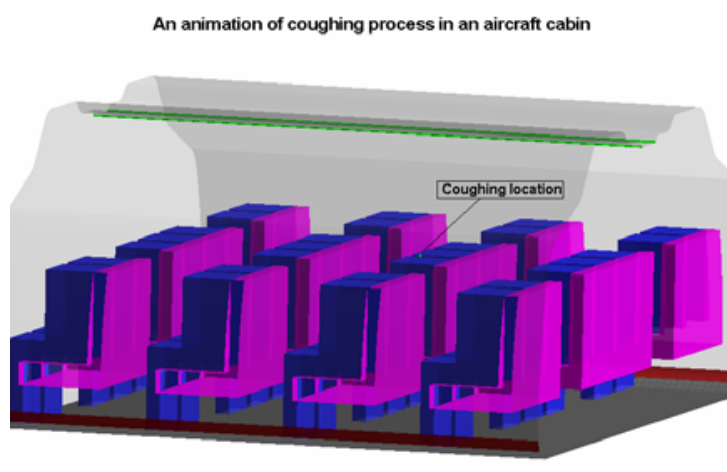

(a) The airliner cabin geometry

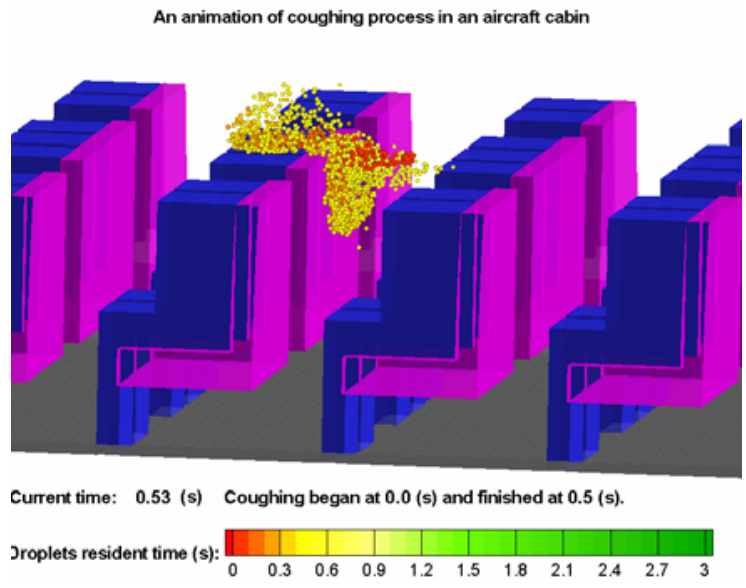

(b) Droplets transport after 0.53 seconds. 


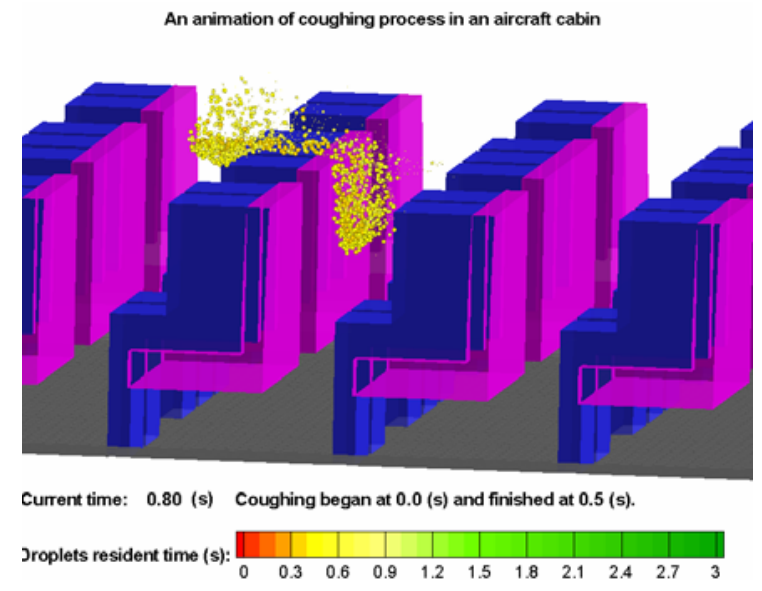

(c) Droplets transport after 0.8 seconds.

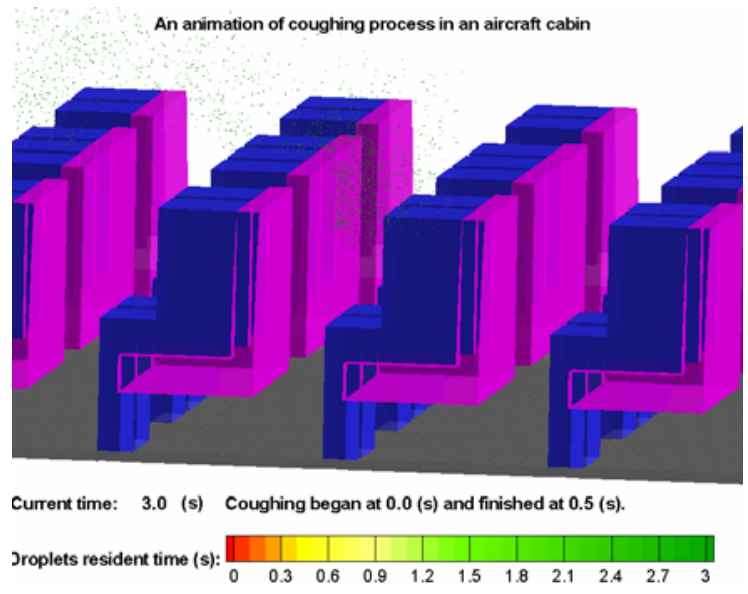

(d) Droplets transport after 3.0 seconds.

Fig. 5. Simulation of droplet dispersion in a Boeing 767 cabin due to coughing of a passenger

Note that liquid droplets can evaporate in air. This is especially evident in cabin air because of the low humidity. In this case, the averaged cabin air temperature was set at $24^{\circ} \mathrm{C}$ and the averaged relative humidity was $15 \%$. The cabin was fully occupied and the ventilation rate was $10 \mathrm{~L} / \mathrm{s}$ per person. The initial velocity of coughing was $10 \mathrm{~m} / \mathrm{s}$ and the coughing last for $0.5 \mathrm{~s}$. The initial droplet diameter was $100 \mu \mathrm{m}$. Each liquid droplet had a non-evaporative nucleus of $1 \mu \mathrm{m}$. Although the actual simulation was performed for four rows of seats, period boundary conditions were applied so one could think the simulation was actually for an infinitely long cabin. Figures 5(b), 5(c), and 5(d) show temporal distributions of the liquid droplets and their sizes at $0.053 \mathrm{~s}, 0.8 \mathrm{~s}$, and $3.0 \mathrm{~s}$, respectively. The simulated results clearly show that the particle could be dispersed to several rows in front of the coughing passenger. In Fig. 5, red dots were for large size of particles, yellow for moderate size of particles and green for nuclei. The liquid in the particles evaporated within three seconds. Although we have no experimental data available to validate the results, they look plausible.

\subsection{Application of LES with Lagrangian method to particle dispersion in a small building}

As LES always computes time-dependent flow even though the flow could be steady state and the accuracy of LES depends on grid resolution, it normally requires one magnitude longer computing time and larger computer memory than RANS modeling. Thus, most of the applications of LES were limited in enclosed environment. In fact, LES with a particle model can be used to study a reasonably sized building.

This section shows the results using the LES with the Lagrangian method for the prediction of particle dispersion in a small building with six rooms as shown in Fig. 6. The particle source was on one end of the corridor and the type of particles is corn-type, which has a diameter of $87 \mu \mathrm{m}$, and a density of $1000 \mathrm{~kg} / \mathrm{m}^{3}$. For this type of particle, the gravity force plays an important role. However, if the gravity force were considered in the current calculation, the particles would not travel through the whole building before falling to the ground. Therefore, we simulated particle transport in the building without gravity. 


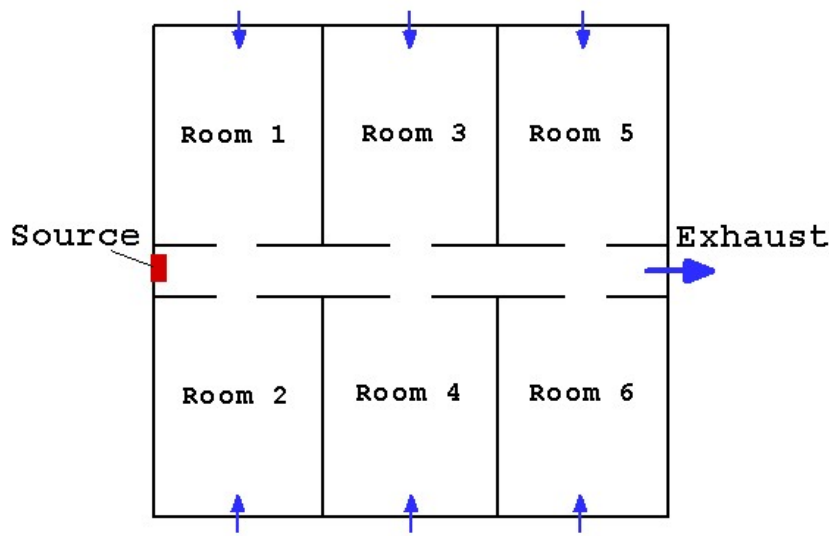

Fig. 6. The floor plan of a six-room building

One thousand corn-type particles were injected into the corridor. They were dispersed into different rooms with time, and some of them were removed through the exhaust. Figure 7 shows the trajectories of these particles after four minutes. It is interesting to note that the particles could enter the rooms although the global flow was one way from the rooms to the corridor. The numerical simulation provided a great insight of flow and particle transport in the building.

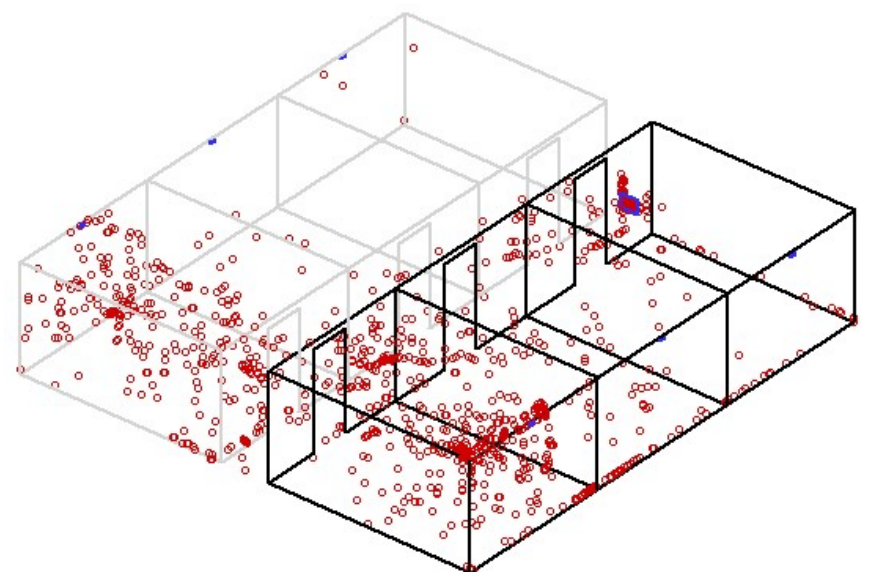

Fig. 7. Trajectories of 1000 particles in the building four minutes after they were injected

\section{Conclusions}

This paper provided an overview of computing particle transport in enclosed environment. The study leads to the following general conclusions:

Both RANS modeling and LES have been used to predict airflow in rooms. The computed results were in reasonably agreement with the measured distributions of air velocity, air temperature, and tracer-gas concentration. The performance of RANS modeling was similar to that of LES for the cases studied. However, LES provides more detailed information than RANS modeling and uses only one or no empirical coeffient. LES is a powerful tool for multiple scale studies.

This investigation used the Lagrangian and Eulerian methods to determine particle transport in rooms and compared the computed results with the corresponding experimental data. Again, the study found that the two methods could predict the particle distributions with acceptable accuracy and the two methods performed similarly. But, the Lagrangian method can track every particle and does not have an empirical diffusion coefficient. It is superior to the Eulerian method that gives only global value for multiple-scale studies.

The Lagrangian method was further used with the RANS model for predicting liquid droplet dispersion in a Boeing 767 cabin, and with the LES for solid particle dispersion in a small building with six rooms. The computed results provided very informative and useful information. 


\section{Acknowledgements}

The authors would like to thank Dr. Mingde Su and Dr. Yi Jiang for their contributions to the results published in this paper. This research is funded partially by Chinese Academy of Sciences and partially by the U.S. Federal Aviation Administration (FAA) through the FAA Cooperative Agreement 04-C-ACE-PU, Amendment 002 for the Air Transport Center of Excellence for Airliner Cabin Environment Research (ACER).

\section{Nomenclature}

\begin{tabular}{|c|c|}
\hline $\mathrm{C}$ & particle concentration \\
\hline $\mathrm{C}_{\mathrm{c}}$ & Cunningham correction factor \\
\hline$C_{D}$ & drag coefficient \\
\hline $\mathrm{C}_{S G S}$ & Smagorinsky constant \\
\hline $\mathrm{C}_{1 \varepsilon}, \mathrm{C}_{2 \varepsilon}, \mathrm{C}_{3 \varepsilon}$ & turbulent model coefficients \\
\hline $\mathrm{C}_{\mu}$ & turbulence model coefficient \\
\hline$D^{\mu}$ & Brownian diffusivity of particles \\
\hline$d_{p}$ & particle diameter \\
\hline$f_{p}$ & response frequency of particle \\
\hline $\mathrm{F}_{\mathrm{bi}}$ & component of the Brownian force in the $x_{i}$ direction \\
\hline $\mathrm{F}_{\text {DRAG i }}$ & component of the drag force in the $x_{i}$ direction \\
\hline $\begin{array}{l}\mathrm{F}_{\text {GRAV }} \\
\mathrm{F}_{\mathrm{i}}\end{array}$ & $\begin{array}{l}\text { component of the gravity force in the } x_{i} \text { direction } \\
\text { forces in the } x_{i} \text { direction }\end{array}$ \\
\hline $\mathrm{F}_{\mathrm{SAF} i}$ & component of the Saffman lift force in the $x_{i}$ direction \\
\hline $\mathrm{G}_{\mathrm{b}}$ & buoyant production \\
\hline $\mathrm{G}_{\mathrm{k}}$ & shear production \\
\hline$G\left(x, x^{\prime}\right)$ & the filter kernel \\
\hline & $\begin{array}{l}\text { component of the gravitational acceleration in the } x_{i} \text { direction } \\
\text { turbulence kinetic energy }\end{array}$ \\
\hline$m_{P}$ & particle mass \\
\hline p & air pressure \\
\hline $\operatorname{Re}_{\mathrm{P}}$ & particle Reynolds number \\
\hline Sc & source term of particle concentration equation \\
\hline$S_{i j}$ & strain rate of the tensor \\
\hline $\mathrm{S}_{\mathrm{ui}}$ & source term of momentum equation \\
\hline$U_{i}$ & mean velocity component in the $x_{i}$ direction \\
\hline $\mathrm{U}_{\mathrm{j}}$ & mean velocity component in the $x_{j}$ direction \\
\hline $\overrightarrow{\mathrm{u}}$ & fluid velocity \\
\hline u' & root-mean square velocity \\
\hline $\mathrm{u}_{\mathrm{i}}$ & component of filtered fluid velocity in the $x_{i}$ direction \\
\hline$u_{i}^{\prime}$ & modeled fluctuation velocity of air in the $x_{i}$ direction \\
\hline$u_{j}$ & component of filtered fluid velocity in the $x_{j}$ direction \\
\hline $\overrightarrow{\mathrm{V}}$ & particle velocity \\
\hline$v_{i}$ & component of particle velocity in the $x_{i}$ direction \\
\hline$X_{i}$ & coordinate of particle in i direction \\
\hline$x_{i}$ & coordinate in i direction \\
\hline $\mathrm{x}_{\mathrm{j}}$ & coordinate in j direction \\
\hline$Y(t)$ & lateral particle displacement \\
\hline
\end{tabular}

Greek symbols

$\begin{array}{ll}\Gamma & \text { effective particle diffusion coefficient } \\ \Delta_{\mathrm{i}} & \text { filter width } \\ \Delta_{\min } & \text { minimum grid space } \\ \Delta \mathrm{t} & \text { the time step } \\ \varepsilon & \text { dissipation rate of turbulence kinetic energy } \\ \zeta & \text { Gaussian random number } \\ v & \text { fluid kinetic viscosity }\end{array}$




$\begin{array}{ll}v_{\text {eff }} & \text { effective kinetic viscosity } \\ v_{p} & \text { turbulent diffusivity of particles } \\ v_{t} & \text { turbulent viscosity } \\ \rho & \text { air density } \\ \rho_{p} & \text { particle density } \\ \rho_{r} & \text { reference air density } \\ \sigma & \text { Boltzmann constant } \\ \sigma_{k} & \text { turbulent Prandtl number for } \mathrm{k} \\ \sigma_{T} & \text { turbulent Prandtl number for temperature } \\ \sigma_{\varepsilon} & \text { turbulent Prandtl number for } \varepsilon \\ \tau_{i j} & \text { subgrid-scale Reynolds stresses } \\ \tau_{t} & \text { turbulent Reynolds stresses } \\ v_{S G S} & \text { subgrid-scale eddy viscosity }\end{array}$

\section{References}

ASHRAE. (2005). ASHRAE Handbook - Fundamentals. Atlanta: ASHRAE.

Crowe, C., Sommerfeld, M. \& Tsuji, Y. (1998). Multiphase Flows with Droplets and Particles, Boca Raton, FL: CRC Press.

Deardorff, J.W. (1970). A numerical study of three-dimensional turbulent channel flow at large Reynolds numbers. J. Fluid Mech. 41, 453-480.

Hinds, W.C. (1982). Aerosol Technology: Properties, Behaviour, and Measurement of Airborne Particles, John Wiley and Sons, Inc.

Hinze, J.O. (1975). Turbulence, $2^{\text {nd }}$ Edition. New York: McGraw Hill.

Hjelmfelt, A.T. \& Mockros, L.F. (1966). Motion of discrete particles in a turbulent fluid. App. Sci. Res., $16,149$.

Lai, A.C.K. \& Nazaroff, W.W. (2000). Modeling indoor particle deposition from turbulent flow onto smooth surfaces. J. Aerosol Sci. 31, 463-476.

Launder, B.E. \& Spalding, D.B. (1974). The numerical computation of turbulent flows. Computer Methods in Applied Mechanics. (3: pp. 269-289).

Li, A. \& Ahmadi, G. (1992). Dispersion and deposition of spherical particles from point sources in a turbulent channel flow. Aerosol Science and Technology, 16, 209-226.

Loth, E. (2000). Numerical approaches for motion of dispersed particles, droplets and bubbles, Progress in Energy and Combustion Science. 26, 161-223.

McLaughlin, J.B. (1989). Aerosol deposition in a numerically simulated channel flow. Physics of Fluids A. $1(7), 1211-1224$.

Patankar, S.V. (1980). Numerical Heat Transfer and Fluid Flow. Washington, DC: Hemisphere.

Saffman, P.G. (1965). The lift of a sphere in a slow shear flow. Journal of Fluid Mechanics. 22 (2), 385-400.

Smagorinsky, J. (1963). General circulation experiments with the primitive equations. I. The basic experiment. Monthly Weather Review, 91, 99-164.

Wallace, L. (1996). Indoor Particles: a review. Journal of the Air \& Waste Management Association, 46(2), 98-126.

Yuan, X., Chen, Q., Glicksman, L. R., Hu, Y., \& Yang, X. (1999). Measurements and computations of room airflow with displacement ventilation. ASHRAE Transactions, 105(1), 340-352.

Zhang, Z. \& Chen, Q. (2005). Experimental measurements and numerical simulations of fine particle transport and distribution in ventilated rooms. In preparation.

Zhao, B., Zhang, Z. Li, X. \& Huang, D. (2004). Comparison of diffusion characteristics of aerosol particles in different ventilated rooms by numerical method. ASHRAE Transactions, 110, 88-95. 